\title{
Generisches Simulationsmodell für automatische Hochregallagersysteme
}

\author{
Walter Wincheringer, Marko Sekulic, Marec Kexel \\ Digitales Produktionslabor, Hochschule Koblenz, Konrad-Zuse-Str. 1, 56075 Koblenz, Germany; \\ dpl@hs-koblenz.de; wincheringer@hs-koblenz.de; sekulic@hs-koblenz.de; mkexel@hs-koblenz.de;
}

\begin{abstract}
Zusammenfassung. Der Einsatz von automatischen Hochregallagern (aHRL) hat sich seit Jahrzehnten in der Praxis bewährt. Eine Auslegung bzgl. der Leistung und des Durchsatzes erfolgt meist mit Hilfe von empirischen Näherungslösungen gemäß der FEM 9.851 [1] oder der VDI 3561 [2]. Für eine realistische Leistungsbetrachtung und für Systeme, die nicht durch diese Richtlinien abgedeckt sind, bedarf es jedoch einer zeitdiskreten Simulation (discrete event simulation, DES) des Lagersystems. Der Aufwand zur Durchführung einer Simulationsstudie, inkl. dem Aufbau eines Simulationsmodells, wird mit 1,5 - 6 Monaten bemessen [3][4]. Eine Weiterentwicklung von spezifischen Referenzmodellen hin zu einem parametrierbaren generischen Simulationsmodell ist ein vielversprechender Lösungsansatz und reduziert den Aufwand erheblich. Die Entwicklung und Anwendung eines generischen Simulationsmodells für aHRL, sowie die damit verbundenen Vorteile, werden im Beitrag erläutert.
\end{abstract}

\section{Ausgangsituation}

Automatische Lagersysteme, wozu automatische Hochregallagersysteme (aHRL) gehören, sind in der Praxis stark verbreitet und übernehmen bei der Lagerhaltung in Industrie und Handel eine wichtige Rolle. Der Markt für automatische Lagersysteme in Europa wächst jährlich um ca. 9,5 Prozent und umfasst ca. 1,9 Mrd. Euro [5].

Daher gibt es ein breites Spektrum an aHRL-Herstellern, die unterschiedliche Lösungskonzepte im Markt anbieten. Zur Auslegung und Leistungsberechnung wird häufig auf Erfahrungswerte und Normen zurückgegriffen, wie die FEM 9.851, VDI 3561 sowie VDI 4480 [6]. Insbesondere die techn. Auslegung des Trägerfahrzeuges (z.B. Regalbediengerät, RBG), dessen Beschleunigungen und Geschwindigkeiten in der Horizontalen und Vertikalen, die Lagergeometrie (Gesamtlänge, -höhe, Lagerfachhöhe und Lagerplatzabstände), sowie die Lagerstrategie (Art und Umfang des Arbeitsspiels,
Ein-, Auslagerspiel, Lagerplatzvergabe und Zugriff auf Ladeeinheiten), bestimmen die Ein- und Auslagerungsleistung eines aHRL. Die maximal mögliche Durchsatzleistung (Summe der Ein- und Auslagerungen pro Zeiteinheit) wird hierbei häufig als zentrale Kenngröße definiert. Bei der Auslegung basiert diese auf statischen Betrachtungen und mittleren Spielzeiten für Ein- und Auslagerungen auf Basis von Referenzlagerplätzen.

Die Richtlinien haben nur eine eingeschränkte Tauglichkeit, da sie auf Annahmen basieren, wie z.B. chaotischer Einlagerstrategie, und nur für bestimmte Lagerkonfigurationen (i.d.R. einfachtiefe Lagerung) eine Gültigkeit besitzen. Sie sollten nur für eine näherungsweise Bestimmung der Leistung herangezogen werden, da die tatsächliche Leistung in der Praxis davon z.T. erheblich abweicht [7]. Die Anzahl der Doppelspiele, welche von der zeitlichen Abfolge der Ein- und Auslagerungsaufträgen sowie der Bewegungsstrategie abhängt, kann ggf. nicht mit der Annahme aus der Planungsphase übereinstimmen oder die auszulagernden Ladeeinheiten wurden an ungünstigen Positionen im aHRL eingelagert (z.B. bei einem hohen Füllungsgrad des Lagers). Die negativen Auswirkungen können zu erheblichen Produktivitätsverlusten (Rückstau der Fertigware in die Produktion, Produktionsstillstände, mangelnde Versand-, Verladeleistungen) in unterschiedlichen Bereichen führen.

Um diese Probleme zu vermeiden, ist die Durchführung einer ereignisdiskreten Simulation des aHRL notwendig [7]. Eine derartige Simulationsstudie, nach VDI 3633, ist jedoch mit einem erheblichen Aufwand für die Erstellung eines validierten Simulationsmodells verbunden und erfordert die Verfügbarkeit eines Simulationsexperten [8]. Um diesen Aufwand zu reduzieren, haben einige Hersteller in der Vergangenheit sogenannte Referenzmodelle erstellt, die eine spezifische Abbildung ihrer aHRL-Systeme ermöglichen [9]. Die in den Refer- 
enzmodellen nicht abgebildeten Funktionen bzw. Bausteine müssen durch eine manuelle Programmierung aufwendig implementiert werden.

Die Simulation von aHRL ist jedoch nicht nur in der Planungsphase sinnvoll, sondern auch in der Betriebsphase, z.B. bei veränderten Anforderungen durch den Betreiber. Diese können unter anderem die Betriebszeiten (Ein-, Auslagerzeiträume), Anzahl an lagerhaltigen Artikeln (SKU, meist steigend), als auch die Art der Lagerplatzvergabe und den Zugriff auf Ladeeinheiten betreffen. Daher müssen ebenfalls die unterschiedlichen Lagerstrategien bei der Simulation berücksichtigt werden.

\section{Lösungsansatz}

Mit der zunehmenden Digitalisierung der Geschäftsprozesse, der Produkte und der Produktion (Smart Factory) ist die Nutzung von Digitalen Zwillingen (digital twin, DT) stärker in den Fokus gerückt. Sie ermöglichen eine Überprüfung von Konzepten und Ideen in der virtuellen Welt, ohne Einfluss auf das reale System zu nehmen [10]. Übertragen auf aHRL besteht die Zielsetzung darin, einen DT als Simulationsmodell zu erzeugen, um Veränderungen der Anforderungen oder eine Neuauslegung als mögliche "Was-wäre-wenn-Szenarien” zu simulieren und zu evaluieren.

Der DT soll jedoch nicht in einer Simulationssoftware individuell programmiert werden, sondern soll als Weiterentwicklung von Referenzmodellen, in Form eines parametrierbaren generischen Simulationsmodells, dem Anwender zur Verfügung gestellt werden. Um dies zu realisieren ist es erforderlich, die verschiedenen Lagerkonfigurationen einer aHRL-Auslegung, als auch die jeweiligen Lagerszenarien (Initialbestände, Ein- und Auslagerungsaufträge) über eine Benutzerschnittstelle in die Simulation zu integrieren. Die anwendungspezifische Lagerkonfiguration und das -szenario dienen als Eingangsdaten für das generische Simulationsmodell (siehe Abbildung 1).

Aus den Daten und Parametern der Lagerkonfiguration, die über eine Schnittstelle eingegeben (Excel-Template) oder ggf. automatisch von einem ERP, PLM oder CAD-System übernommen wurden, erfolgt die automatische Generierung des Simulationsmodells in Sekundenschnelle. Dadurch ist die Nachbildung einer Vielzahl an unterschiedlichen aHRL-Modellen möglich (siehe Kapitel 3.1).

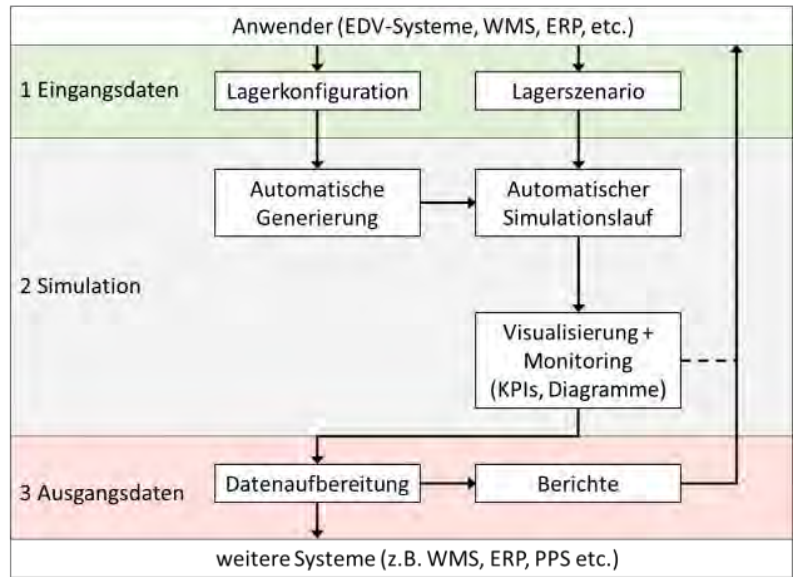

Abbildung 1: Funktionsweise des Simulationsmodells

Lauffähig wird das Simulationsmodell über eine weitere Schnittstelle. Diese lädt die Initialbestände für den Simulationsstart (anfängliche Lagerbelegung, bestehender Auftragsbestand, etc.) des Lagersystems und die Auftragsdaten. Die Auftragsdaten beschreiben das geplante Ein- und Auslagerszenario des aHRL und können von einem Warehouse Management Systems (WMS) bzw. ERP-System importiert bzw. extrapoliert oder von einem Auftragsgenerator erstellt werden [11]. Letzteres ist notwendig, wenn das zu betrachtende Lagerszenario in der Zukunft liegt und auf prognostizierte Lagerbestände und veränderte Auftragsdaten aufbaut (siehe Kapitel 3.2). Anschließend kann der Simulationslauf gestartet und die Aufträge im Lagersimulationssystems durchgespielt werden.

Über eine Darstellung der Ergebnisse, parallel zum Simulationslauf, mittels geeigneter Diagramme und KeyPerformance-Indicators (KPI) können die Simulationsergebnisse dynamisch visualisiert und vom Anwender überprüft werden. Die Ergebnisse können für die Erstellung von Berichten und weiterer Verwendungen exportiert werden.

Da das generische Simulationsmodell für aHRL-Systeme bereits verifiziert und validiert ist, bedarf es keiner Überprüfung des Simulationsmodells. Zur Eingabe der Daten und zur Generierung des Simulationsmodells bedarf es ebenso keiner simulationsspezifischen Programmierarbeit. Somit ist kein Simulationsexperte erforderlich. Jedoch sollte die Anwendung des Tools durch einen Lagersystemfachmann erfolgen, da er die jeweiligen $\mathrm{Pa}$ rameter schnell und einfach zuordnen kann.

Für die Optimierung bestehender oder die Auslegung neugeplanter Lagersysteme ist eine Variation der Ein- 
gangsparameter (Simulationsexperimente) empfehlenswert, um "Was-wäre-wenn-Szenarien” zu generieren, damit ein optimales Lager nach den Vorstellungen und Restriktionenen des Nutzers gefunden werden kann. Zusätzlich sollte durch Simulationsexperimente der Einfluss des Zufalls (in Bezug auf techn. Störungen des Trägerfahrzeuges oder bei einer chaotischen Einlagerung) auf die Ergebnisse bestimmt werden, über eine Variation der Zufallszahlenreihen, um Fehlentscheidungen bzgl. der Neuauslegung zu vermeiden.

\section{Eingangsdaten}

\subsection{Lagerkonfiguration}

Als Datengrundlage dient die Lagerkonfiguration, die das Lagersystem (bau-)technisch und ablauforganisatorisch definieren. Folgende Größen sind variabel über eine Schnittstelle (z.B. Excel-Template) einstellbar:

Tabelle 1: Eingabeparameter zur Lagerkonfiguration

\begin{tabular}{ll}
\hline Lagerkonfiguration & Größen \\
\hline Technik & $\begin{array}{l}\text { Regaldimensionen, Fächeranzahl, } \\
\text {-größe und -tiefe } \\
\text { Anzahl und Position der Bereitstell- } \\
\text { plätze }\end{array}$ \\
\hline Trägerfahrzeug & Typ (RBG, Shuttle) \\
& Lastaufnahmemittel, \\
& Maximale Geschwindigkeiten in x-, y-, \\
& (z)-Richtung mit/ohne Last, \\
& Beschl. und Verzögerungen in x-, y-, \\
(z)-Richtung mit/ohne Last, \\
Konstantzeiten (Transfer, Mastaus- \\
schwingung, Feinpositionierung), \\
Ausfallverhalten, etc. \\
$\begin{array}{l}\text { Bewegungs-, Einlagerungs-, Auslage- } \\
\text { rungs-, Ruhepositions-, Umlagerungs- } \\
\text { strategie, etc. }\end{array}$ \\
\hline Strategien
\end{tabular}

Die Konfiguration der Regale beinhaltet die Angabe der Anzahl der Regalfächer mit der Fachteilung, -ebenen und -tiefe samt den Abmessungen. Daraus werden die einzelnen Lagerplatzpositionen berechnet, sofern eine Homogenität der Regalfächer bzw. - wände gegeben ist [12]. Alternativ können die Lagerplatzpositionen eingegeben werden.

Die Anzahl der Ein- und Auslagerungsbereitstellplätze sowie deren Position können frei variiert werden. Bereitstellplätze können auch innerhalb einer Regalwand platziert werden, wie in der folgenden Abbildung 2 rechts ersichtlich. Hierbei sind die Einlagerungsbereitstellplätze grün, die Auslagerungsbereitstellplätze rot dargestellt.

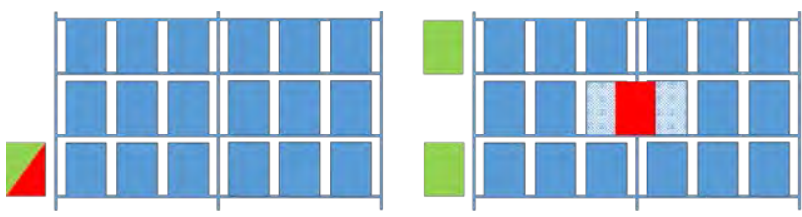

Abbildung 2: Skizze unterschiedlicher Anzahl und Anordnungen der Bereitstellplätze (Seitenansicht)

Neben Regalbediengeräten, welche zeitlich parallel horizontal und vertikal fahren, sind auch Verschiebewagen bzw. Shuttles einstellbar. Diese Fahrzeuge fahren ausschließlich horizontal. Die Vertikalbewegung der Fahrzeuge bzw. der Ladeeinheiten findet hierbei über Lifte statt.

Für die Fahrtzeitberechnung der einzelnen Arbeitsspiele sind die Fahrparameter: Maximale Geschwindigkeit, Beschleunigungen und Verzögerungen relevant. Für die Genauigkeit der Fahrtzeitberechnung werden die Größen hinsichtlich horizontal bzw. vertikal und beladen bzw. unbeladen unterschieden. Bei kurzen Fahrten kann die maximale Geschwindigkeit ggf. nicht erreicht werden, dabei kommt es zu einem dreieckigen Geschwindigkeitsverlauf (auch Dreiecks- bzw. Spitzfahrten genannt) [13].

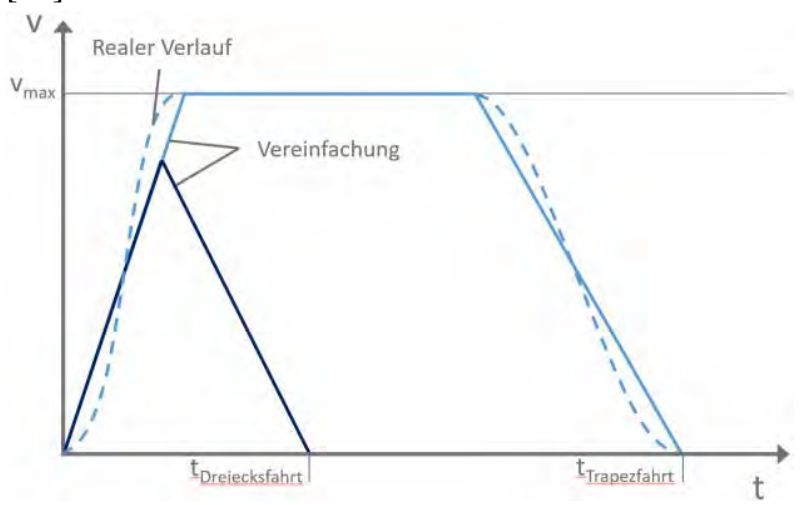

Abbildung 3: v-t-Diagramm von Fahrbewegungen

Bei Regalbediengeräten setzt sich die Diagonalbewegung aus beiden parallelen Bewegungen des Fahrund Hubwerks zusammen. Somit beträgt die Fahrzeit für die Diagonalfahrt:

$$
t_{x, y}=\max \left(t_{x}, t_{y}\right)
$$

Für die Berechnung der Arbeitsspiele werden zusätzlich Verweilzeiten hinzugerechnet. Diese bestehen aus den 
Fahrtbewegungen des Lastaufnahmemittels in z-Richtung (Gabelspielzeit bzw. Transfer der Ladeeinheit) mit den Zeiten für die Feinpositionierung bzw. der Mastausschwingung und evtl. anfallenden Tot- bzw. Schaltzeiten.

Die realtitätsgetreue Abbildung des Lagersystems in einem Simulationsmodell bedingt zusätzlich die stochastisch auftretenden Störungen des Trägerfahrzeugs. Hierbei werden Mean Time Between Failures (MTBF) und Mean Time To Repair (MTTR) verwendet [14]. Diese können nach vorgegebenen Verteilungsfunktionen schwanken. Bei unbekannten Stördaten eignet sich die negative Exponentialverteilung für die Initiierung der Störzeitpunkte und die Erlang-k-Verteilung mit $\mathrm{k}=2$ für die Reparaturdauer [15]. Bei bekannten Ausfallverhalten können benutzerdefinierte Verteilungsfunktionen angegeben werden.

Die Positionen, die bei den Arbeitsspielen angefahren werden, werden anhand der gewählten Lagerbewirtschaftsungsstrategie generiert. Die Bewegungsstrategie entscheidet, ob Einzelsspiele (eine Ein- oder Auslagerung pro Fahrauftrag), Doppelspiele (eine Einlagerung und Auslagerung pro Fahrauftrag) oder Mehrfachspiele (nur bei einem Mehrfach-Lastaufnahemittel möglich) gefahren werden können.

Wo konkret die Ladeeinheiten im Regal eingelagert werden, bestimmt die Einlagerungsstrategie, auch Belegungsstrategie genannt. Hierbei können verschiedene Strategien innerhalb folgender Kategorien eingestellt werden:

- Frei (z.B. chaotisch oder kürzester Fahrweg),

- Fest (durch Vorgabe durch den WMS)

- Zoniert (z.B. ABC-Zonierung)

- Artikel- bzw. chargenrein bei Mehrfachttiefe, etc.

Die Auslagerungsstrategien bestimmen dagegen den Ort bzw. die Ladeeinheit, welche zu einem bestimmten Zeitpunkt ausgelagert wird. Hierbei kann FIFO (bzw. schwaches FIFO bei Mehrfachttiefe [16]) und manuell (Vorgabe durch WMS) unterschieden werden.

Neben diesen Strategien können weitere Strategien, wie z.B. Nichtbeschäftigungs- (Umlagerung von Ladeeinheiten in die Nähe des Auslagerungsbereitstellplatzes oder Mischkanäle verringern) bzw. Umlagerstrategien zur Anwendung kommen.

\subsection{Lagerszenario}

Nachdem das Lagersystem für das Simulationsmodell technisch und ablauforganisatorisch definiert wurde, muss der Initialbestand sowie die Auftragslast für den Simulationslauf bestimmt werden. Hierunter sind unter anderem folgende Daten zu verstehen:

- Zeitpunkt der Auftragsentstehung (Ein- und Auslagerungsauftrag)

- Artikelbezeichung und Attribute:

- Klassifizierung für Zonierung

- Chargennummer

- Ladehilfsmittel, etc.

- Ort der Ein- bzw. Ausschleusung (Zuweisung zu den Bereitstellplätzen)

- Lagerplatz (bei der festen Lagerplatzzuordnung bzw. manuellen Auslagerung)

Die Initialdaten sind ähnlich aufgebaut. Nicht benötigt werden die Angaben zum Zeitpunkt der Auftragsentstehung sowie der Ort der Ein- bzw. Ausschleusung, da die Ladeeinheiten nicht eingelagert werden, sondern initial zum Simulationszeitpunkt Null im Lager vorhanden sind.

Die Daten für das Lagerszenario können bei einem bestehenden Lagersystem aus der WMS-Auftragshistorie (z.B. Auftragsdaten einer gewählten Periode) erstellt werden, wenn für den simulierten Zeitraum eine ähnliche Auftragslast angenommen werden kann. Wenn das nicht der Fall ist, z.B. bei einer veränderten Einlagerung bzw. Auslagerungsstruktur oder einer Neuplanung bei fehlender Auftragshistorie, gibt es zwei Möglichkeiten diese Daten zu erhalten:

- Extrapolation vorhandener WMS-Daten,

- Auftragsdatengenerator.

Eine Extrapolation vorhandener WMS-Daten kommt infrage, wenn zum einen die WMS-Daten verfügbar sind und zum anderen die Auftragsstruktur nicht grundlegend zum Simulationszeitraum verschieden sind. Wenn z.B. neue Artikel hinzukommen und/oder das Einlagerungsund Abrufverhalten stark abweicht, empfiehlt sich ein Auftragsdatengenerator.

\section{Simulation}

\subsection{Automatische Generierung}

Auf Basis der Eingangsdaten (3.1) wird im nächsten Schritt das Simulationsmodell des aHRL automatisch generiert. Es wird eine aufgeklappte Gasse des Regalsystems dargestellt.

In Abbildung 4 ist ein einfachtiefes aHRL generiert worden, welches fünf Ebenen hoch und 7 Regalfächer à 3 Lagerplätze lang ist. Es sind die Bereitstellplätze (grün: Einlagerung; rot: Auslagerung) sowie das Trägerfahrzeug zu sehen. Die Lagerplätze werden durch die Zahlen repräsentiert. In diesem Zusammenhang steht ein 
Lagerplatz mit dem Wert "0" für einen freien Platz. In der Gasse zwischen den beiden Regalwänden ist das Trägerfahrzeug platziert. Auf dem Trägerfahrzeug sind zwei Zahlen ersichtlich, welche die Position in der Höhe darstellt: Die linke Zahl steht für die Start-Ebene, die Rechte für die Ziel-Ebene (zum Start ist kein Ziel vorhanden: 0).

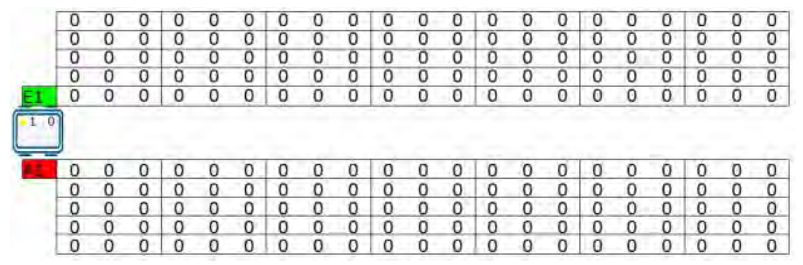

Abbildung 4: Beispielhafte Generierung eines aHRL

Zum Vergleich ist in Abbildung 5 ein weiteres aHRL mit anderen Abmessungen (7 Ebenen, 8 Regalfächer) und unterschiedlichen Positionen der Bereitstellplätze abgebildet. Ersichtlich wird, dass Bereitstellplätze in der Regalwandmitte entsprechende Lagerplätze blockieren (vgl. Abbildung 2).

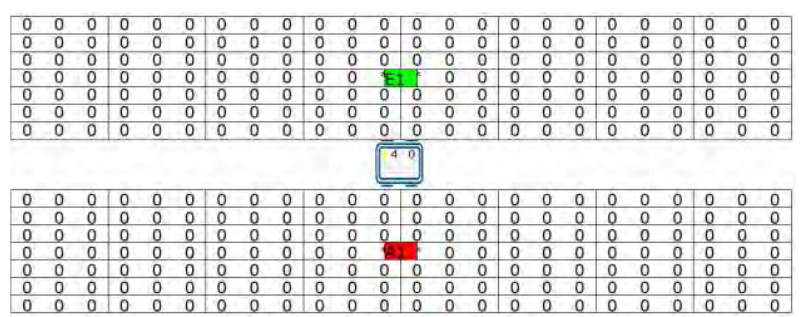

Abbildung 5: Bsp. Generierung eines aHRL mit veränderter Geometrie und Bereitstellpositionen

\subsection{Automatischer Simulationslauf}

Der automatische Simulationslauf beginnt nach der Generierung des Simulationsmodells. Im ersten Schritt werden die Initialdaten eingelesen, welche die Ladeeinheiten zu Beginn platzieren, repräsentiert durch eine „1“, wie in Abbildung 6 ersichtlich:

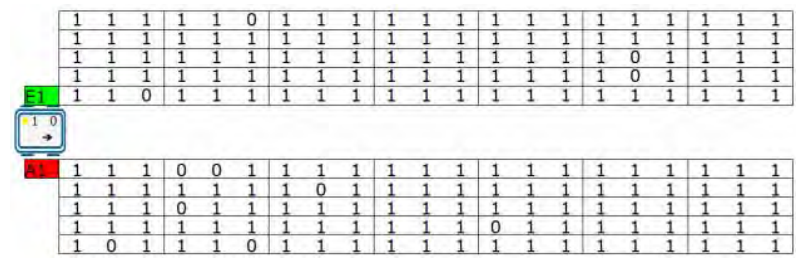

Abbildung 6: Beispielhafte Startbefüllung

Der Simulationszeitraum wird dabei anhand der Daten des Lagerszenarios bestimmt. Wenn entsprechende Auftragsdaten für 3 Monate vorliegen, so beträgt auch der Simulationszeitraum 3 Monate. Die Simulationsdauer beträgt hingegen, je nach Simulationsgeschwindigkeit, Sekunden bis Minuten. Der Ablauf folgt dabei der ausgewählten Lagerstrategie sowie der hinterlegten Ein- und Auslagerungsaufträge. Das Fahrzeug fährt anhand dieser Vorgaben die entsprechenden Positionen in der Regalwand an und führt die geforderten Aktionen aus. Dabei werden die Zeiten für eine Aktion durch die hinterlegten Parameter exakt berechnet.

Ein Beispiel für ein Einlagerungsspiel ist in der Abbildung 7 ersichtlich und läuft wie folgt ab:

1. Ankunft einer Ladeeinheit am Bereitstellplatz und Auftragsannahme durch das Trägerfahrzeug.

2. Aufnahme der Ladeeinheit.

3. Fahrt zum Lagerplatz, gemäß der Einlagerungsstrategie.

4. Abgabe der Ladeeinheit an den Lagerplatz.

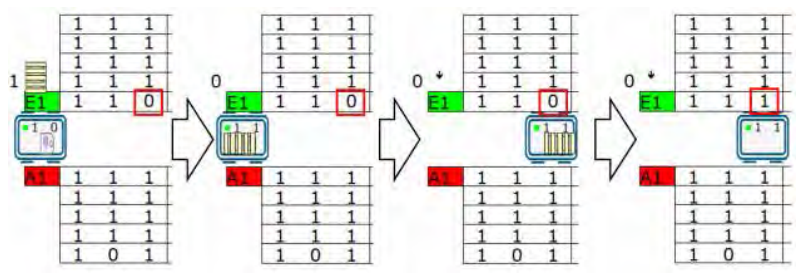

Abbildung 7: Beispiel für ein Einlagerungsspiel

Die Auftragssstruktur ist mitentscheidend für die tatsächliche Leistung eines Lagersystems, da anhand der Aufträge im Simulationsmodell entschieden wird, wie die Fahrten des Trägerfahrzeugs ausgeführt werden, z.B. ob Einzel-, Doppel- oder Mehrfachspiele gefahren werden. Diese Einflussgröße wird, wie in Kapitel 1 beschrieben, nicht in den mathematisch-analytischen Berechnungsmethoden z.B. der FEM 9.851 oder VDI 3561 betrachtet. Es wird lediglich die mittlere Spielzeit für ein Einzelspiel bzw. für ein Doppelspiel bestimmt. Wieviele Doppelspiele tatsächlich pro Zeiteinheit gefahren werden können, ist nur durch die Simulation zu bestimmen.

\subsection{Visualisierung, Monitoring und Ausgangsdaten}

Parallel zum Simulationlauf werden die unterschiedlichsten Diagramme dynamisch dargestellt und KPIs aufgezeichnet. So werden unter anderem die Auslastung des Trägerfahrzeugs in variablen Zeitintervallen aufgezeichnet (siehe Abbildung 8) sowie die Lagerauslastung über die Zeit (siehe Abbildung 9). 


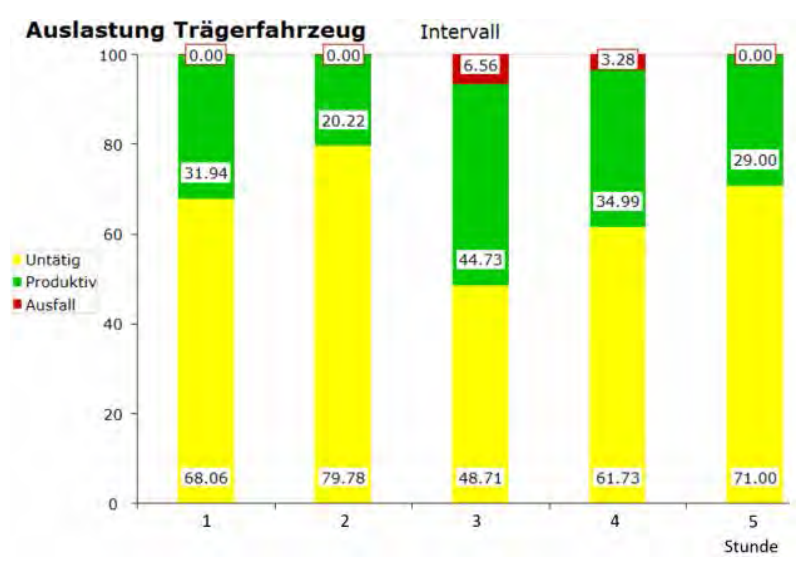

Abbildung 8: Beispielhafte Auslastung des Trägerfahrzeugs im Stundenintervall

Bei einem zonierten Lager lassen sich ebenfalls die Auslastung der unterschiedlichen Zonen im Lagerauslastungsdiagramm darstellen. Bei einem mehrfachtiefen Lager lassen sich die voll-, teilbelegten und leeren Kanäle anzeigen.

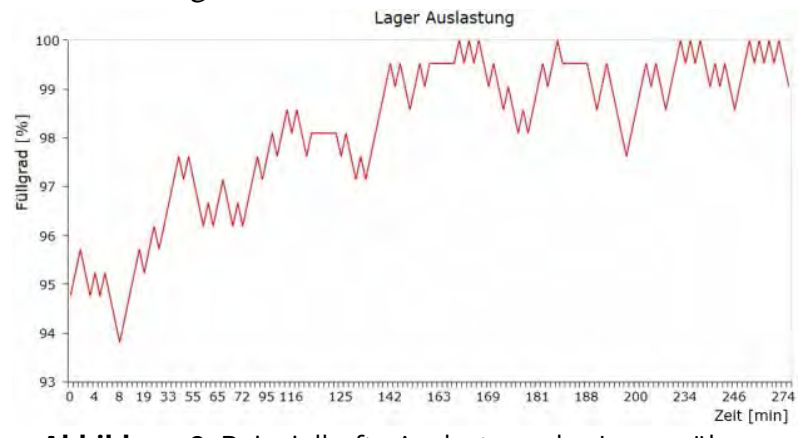

Abbildung 9: Beispielhafte Auslastung des Lagers über die Zeit [min]

Die Diagramme ermöglichen es Lastspitzen über die komplette Simulationsdauer zu identifizieren. Desweiteren werden weitere KPIs wie:

- Durchsatz,

- mittlere Spielzeit,

- Anzahl der Einzel-, Doppel- und Mehrfachspiele,

- Grenzdurchsatz, etc.

über den Simulationslauf hinweg berechnet und dargestellt. Wenn es die Lagerkonfuguration (z.B. Lagergeometrie) zulässt, werden parallel dazu die Ergebnisse der Simulation mit den Ergebnissen der FEM-Berrechnung gegenübergestellt, um so Abweichungen der statischen Berechnung auf einen Blick zu erkennen.

Die Vielzahl und Darstellungsweise der KPIs ermöglichen es dem Lagerplaner und -betreiber Engpässe und Optmierungspotenziale auf einen Blick zu erkennen.
Dabei können die Simulationergebnisse durch die entsprechenden Schnittstellen exportiert und nach der Datenaufbereitung für Reports, Big-Data-Analysen oder für weitere Systeme, wie z.B. WMS, ERP, PPS, als Eingangsdaten weiterverwendet werden.

\begin{tabular}{|c|c|c|c|}
\hline \multicolumn{2}{|l|}{ Lagerkapazität } & \multicolumn{2}{|l|}{ Anzahl der Spiele } \\
\hline Anzahl der Lagerplätze & 210 & Anzahl der Spiele & 86 \\
\hline Belegte Lagerplätze & 209 & Anzahl der Einlagerungsspiele & 30 \\
\hline Blockierte Plätze & 0 & Anzahl der Auslagerungsspiele & 20 \\
\hline Lagerauslastung & 0.995 & Anzahl der Doppelspiele & 36.5 \\
\hline \multirow{4}{*}{\begin{tabular}{|l|} 
Anzahl der Lagerkanäle \\
Leere Kanäle \\
Volle Kanäle \\
Teilbelegte Kanäle \\
Blockierte Kanăle \\
\end{tabular}} & 210 & Anzahl der Zyklen & 124 \\
\hline & $\begin{array}{c}1 \\
209\end{array}$ & \multicolumn{2}{|l|}{ Anteil DS / ES } \\
\hline & $\begin{array}{c}209 \\
0\end{array}$ & Anteil an Doppelspiele & 0.414 \\
\hline & 0 & Anteil an Einzelspiele & 0.586 \\
\hline \multicolumn{2}{|l|}{ Spielzeiten [sec] } & $\begin{array}{l}\text { Anteil an Einlagerungsspiele } \\
\text { Anteil an Auslagerungsspiele }\end{array}$ & $\begin{array}{l}0.356 \\
0.230\end{array}$ \\
\hline $\begin{array}{l}\text { Summe der Spielzeiten } \\
\text { Mittlere Spielzeit }\end{array}$ & \begin{tabular}{c|}
5064 \\
58.88
\end{tabular} & \multicolumn{2}{|l|}{ Durchsatz } \\
\hline Summe der Doppelspielzeiten & 2807 & Gesamt-Durchsatz & 123 \\
\hline Mittlere Doppelspielzeit & 77.15 & Durchsatz pro Std. & 29.25 \\
\hline Summe der Einlagerungsspielzeit & 1347 & Anzahl an Einlagerungen & 67 \\
\hline Summe der Auslagerungsspielzeit & 910 & Anzahl an Auslagerungen & 56 \\
\hline Mittlere Einlagerungsspielzeit & 44.90 & Einlagerungen pro Std. & 15.93 \\
\hline \multirow[t]{5}{*}{ Mittlere Auslagerungsspielzeit } & 45.51 & Auslagerungen pro Std. & 13.62 \\
\hline & & \multicolumn{2}{|l|}{ Grenzdurchsatz } \\
\hline & & Max. Doppelspiele pro Std. & 45.53 \\
\hline & & Max. Einlagungsspiele pro Std. & 80.17 \\
\hline & & Max. Auslagerungsspiele pro Std. & 79.11 \\
\hline
\end{tabular}

Abbildung 10: Ausschnitt aus dem KPI-Dashboard

\section{Erkenntnisse}

Das generische Simulationsmodell für automatische Hochregallager wurde in einer zweijährigen Zusammenarbeit mit einem Industriepartner entwickelt und auf Basis des Softwaretools WITNESS programmiert. Als Schnittstelle wird ein Excel-Template verwendet. Eine ausführliche Validierung mit Real-Daten wurde ausgeführt und das Tool ist seit 2019 im praktischen Einsatz. Es werden sowohl Auslegungen von aHRL-Neuplanungen, als auch Optimierungen von bestehenden Systemen, mit Hilfe des Simulationstools ausgeführt.

Durch die Verwendung des generischen Simulationsmodells entfallen Programmierarbeiten. Somit sind auch Nicht-Simulationsexperten, wie beispielsweise Mitarbeiter im technischen Vertrieb, in der Lage das Simulationstool zu nutzen. Dadurch kann die Simulation bereits in der frühen Angebotsphase eingesetzt werden. Dies führt dazu, dass Sicherheitszuschläge und Leistungsreserven reduziert werden können. Zusätzlich lässt sich der aHRL-Abnahmeprozess vereinfachen, da bereits eine Absicherung der Leistungsdarstellung duch die Simulation erfolgt ist. Weiterhin lassen sich die optimale Lagerkonfiguration und -strategie durch den Einsatz des generischen Simulationsmodells frühzeitig bestimmen und absichern. Die Vorteile werden nachfolgend zusammengefasst: 
- Erhebliche Verringerung des Aufwandes der Simulation (keine Formalisierung und Implementierung bzw. Programmierung, gemäß der VDI 3633, notwendig).

- Durch die Schnittstelle können die benötigten Daten zielgerichtet und schnell erfasst werden.

- Kein Simulationsexperte oder Programmierer erforderlich.

- Nutzung der Simulation in einer frühen Angebotsphase, z.B. durch den technischen Vertrieb.

- Sicherheitszuschläge und Leistungsreserven können reduziert werden.

- Abgesicherte Leistungsdarstellung vereinfacht den aHRL-Abnahmeprozess.

- Frühzeitige Bestimmung und Absicherung einer optimalen Lagerkonfiguration samt -strategie, gemäß dem gewählten Szenario.

- Simulationsexperimente können direkt ausgeführt und -ergebnisse präsentiert werden.

Das generische Simulationsmodell für aHRL wird aktuell für weitere Lagersystemkonfigurationen (u.a. zusätzliche Lagerbewirtschaftungsstrategien) weiterentwickelt.

\section{References}

[1] Fédération Européenne de la Manutention, FEM 9.851: Leistungsnachweis für Regalbediengeräte - Spielzeiten. 2003.

[2] Verein Deutscher Ingenieure, VDI-Richtlinie 3561: Testspiele zum Leistungsvergleich und zur Abnahme von Regalförderzeugen. Düsseldorf: VDI-Verlag; 1973.

[3] Wortmann D. Webinar zum Tag der Logistik SimPlan: Wie die Simulation bei der Planung und Optimierung von Distributionszentren und Lagersystemen hilft. 16.04.2020

[4] Vialog Logistik Beratung. Wann lohnt sich eine Lager-Simulation? In: https://vialog-logistik.com/2015/10/wannlohnt-sich-eine-lager-simulation/. Zugriff am: 14.09.2020. 2015.

[5] MarketsandMarkets. Automated Storage and Retrieval System (ASRS) Market with COVID-19 Impact Analysis by Type (Unit Load, Mini Load, VLM, Carousel, Mid Load), Function (Storage, Order Picking, Assembly, Distribution, Kitting), Industry, and Region- Global Forecast to 2025. 2020.

[6] Verein Deutscher Ingenieure, VDI 4480: Durchsatz von automatischen Lagern mit gassengebundenen Regalbediensystemen, Blatt 1. Berlin: Beuth Verlag; 1998.

[7] Ten Hompel M, Schmidt T, Dregge J. Materialflusssysteme: Förder- und Lagertechnik. 4. Auflage. Berlin: Springer; 2018. S.204.
[8] Verein Deutscher Ingenieure, VDI-3633: Simulation von Logistik-, Materialfluss- und Produktionssystemen Grundlagen, Blatt 1. Berlin: Beuth Verlag; 2014.

[9] Klinger A, Wenzel S. Referenzmodelle - Begriffsbestimmung und Klassifikation. In: Wenzel, S. Referenzmodelle für die Simulation in Produktion und Logistik, Erlangen: SCS; 2000. S. 13 - 29.

[10] Fraunhofer-Institut für Produktionsanlagen und Konstruktionstechnik (IPK). Smarte Fabrik 4.0 - Digitaler Zwilling. Berlin; 2018.

[11] Rabe M, Spieckermann S, Wenzel S. Verifikation und Validierung für die Simulation in Produktion und Logistik: Vorgehensmodell und Techniken. Berlin, Heidelberg: Springer; 2008. S. $89 \mathrm{ff}$.

[12] Atz T. Eine algorithmenbasierte Methode zur ganzheitlichen Systemplanung automatischer Hochregallager. Dissertation, Technische Universität München (TUM); 2016. S.59 f.

[13] Lippolt CR. Spielzeiten in Hochregallagern mit doppeltiefer Lagerung. Dissertation Karlsruher Institut für Technologie (KIT): 2003. S. 58.

[14] Verein Deutscher Ingenieure, VDI 3973: Durchsatz und Spielzeitberechnungen in Stückgut-Fördersystemen. Berlin: Beuth Verlag; 2018.

[15] Gutenschwager K, Rabe M, Spieckermann S, Wenzel S. Simulation Produktion und Logistik: Grundlagen und Anwendungen. Berlin: Springer; 2017. S. 107 f.

[16] Gudehus T: Logistik 2: Netzwerke, Systeme und Lieferketten. 4. Auflage. Berlin, Heidelberg: Springer; 2012. S. 648. 